Robert S. Anderssen, Maureen P. Edwards and Sergiy Pereverzyev Jr.

\title{
9.1 Modeling the Positioning of Trichomes on the Leaves of Plants
}

\begin{abstract}
A continuing and future challenge in plant science is the "genetics of geometry" [3]: the recovery of information about the dynamics of the genetic mechanisms by which plants control the development of various features of their geometry. Some representative publications dealing with such issues include: (i) the modeling of plant architecture using L-systems and rewriting [18], (ii) the genetic control of floral development $[4,10]$, and (iii) the positioning of the trichomes (hairs) on the leaves of plants such as Arabidopsis thaliana [23,25]. It is the positioning of trichomes which is examined in this chapter. The use of reaction-diffusion models is compared with cellular signaling and switching models. It is concluded that, in performing simulations to understand the dynamics of the mechanisms that control pattern formation in plants, it is necessary to work with a cellular model of the plant organ being studied in order to improve on current understanding about how the genetics controls the signaling and switching between cells to produce the observed patterns.
\end{abstract}

Keywords. Genetics of Geometry, Hexagonal Recursion, Leaf, Plant, Reaction-diffusion, Trichome

2010 Mathematics Subject Classification. 35K57, 92C15, 92C80

\subsubsection{Introduction}

The importance of plants (and insects) in the study of the genetics and biology of all organisms relates to the fact that information recovered about the developmental biology of plants can be utilized, through bioinformatics, to improve on current understanding about the developmental biology of non-plants. This is a direct consequence of the revolution in molecular biology that has followed the publication of the double helix interpretation of heredity [33], and the consequential technological revolution associated with full genome sequencing of key organisms (e.g., Arabidopsis), with the discovery and exploitation of gene silencing, and with the fact, being exploited as an essential aspect of bioinformatics, that genes in different organisms with similar DNA sequences are often associated with similar phenotypes and roles. In addition, experimentation with plants is less expensive and ethically more acceptable than with mammals. 
A key example is the genetics of developmental biology. As detailed in the published literature, there has been a two-way street between the genetic studies of the developmental biology of plants and fruit flies [2].

This leads naturally to the study of pattern formation. Biologically, it is important from both theoretical and practical perspectives. For the breeding of plants, there is a need to know which genes control the geometry of plants so that new varieties allow for ease of harvesting and protection against wind damage. Theoretically, any research which yields an enhanced understanding of pattern formation contributes to improving the science of genetics.

An early illustration of the fact that complex biological developmental processes can be simulated using simple algorithms was given by Young [38] in his modeling of the growth of the pea plant. This is consistent with the Wolpert hypothesis [37] that

"It is clear that the egg contains not a description of the adult, but a program for making it, and this program may be simpler than the description. Relatively simple cellular forces can give rise to complex changes in form; it seems simpler to specify how to make complex shapes than to describe them."

The relevance and importance of this observation, especially from a mathematical modeling perspective, is reflected in the fact that any system which is robust can be modeled by the simple model which captures the essence of the phenomenon involved [5]. In addition, the recent research on the crocheting of structures with hyperbolic surfaces [30], of which corals are real-world examples, represents independent validation for the Wolpert hypothesis.

The importance of Young's contribution is that he argued and showed that an appropriate algorithm for the growth of the pea must not only be simple but also be able to generate, with small changes in key parameters, the known mutants. In fact, it represents a generic comment about modeling developmental processes in biology and, indirectly, about modeling pattern formation.

Consequently, in order to illustrate the role of mathematics in the life sciences, the motivation for this paper is an examination of how to construct simple models for the positioning of trichomes on the leaves of plants. Various models have been proposed including

(a) activator-inhibitor reaction-diffusion (AIRD) PDEs models [13],

(b) cellular signaling and switching models [7, 25],

(c) phenomenological genetic models-logical descriptions of the gene activity in terms of diagrammatic models, and

(d) systems of ordinary differential equations which model the known genetics and a biological interpretation of the roles of the genes [6]. 
It is known that, in the positioning of trichomes, not only are there mutants with no or sparsely irregularly spaced trichomes but also ones with random clumping of the trichomes. Consequently, a good test of a model of a developmental process is whether that model can, with a simple change in parameterization, simulate both the wild type and known mutants such as the clumping. Here, the clumping mutant test is chosen as it highlights a clear difference between the various models and gives strong support to the use of the cellular signaling and switching ones.

Comment. As already mentioned, there is an extensive literature which discusses trichome positioning on Arabidopsis leaves where theoretical modeling is matched with experimental details which include the assumed activity of genes known to be involved. The emphasis ranges from the philosophical and experimental to the highly technical. The challenge is the identification of a framework within which logical conclusions can be made about how the genes orchestrate the resulting pattern. It is more than simply saying that such a system can generate patterns and is therefore the mechanism involved. In fact, the situation is sometimes confused because of the failure to draw a clear distinction between the simulation of pattern formation by a complex mathematical model, for which there is no natural biological mechanistic interpretation, and the formation of simple combinatorial algorithms (such as Young's pea growth model) that have a biological mechanistic structure. Digiuni et al. [6] acknowledge the importance of modeling the essential biological mechanism. They propose a coupled theoretical/experimental approach based on an ordinary differential equation model of the time evolution of key genes.

The chapter has been organized in the following manner. The role and limitations of AIRD PDEs in modeling the positioning of the trichomes is examined in Section 9.1.2. Here, their failure in being able, with a small change in parameterization, to generate clumping mutants is explained. These limitations represent motivation for cellular signaling and switching models. The hexagonal recursion implementation [25] is introduced and discussed in Section 9.1.3. Using simulations, it is shown how it can generate, with small changes in the control parameters, the wild type and various mutants including the clumping ones. It is stressed that, for the structure of an algorithm of a developmental process, a minimum requirement must be that mutants can be generated by changing the key parameters that control the development of the wild type. Examples include the turning $\mathrm{ON}$ or $\mathrm{OFF}$ of a parameter to simulate the ON-OFF (or OFF-ON) switching of a key gene, or, as in Young's model of pea development, the accumulating of a signaling molecule (hormone) which controls change from one state to another. The chapter concludes in Section 9.1.4 with a discussion about cellular modeling as a basis for the identification of the dynamics of mechanisms controlling various aspects of pattern formation in plants. 


\subsubsection{Activator-inhibitor Reaction-diffusion Modeling of the Trichome Positioning}

For the modeling of the positioning of trichomes (hairs) on the leaves of plants (in particular Arabidopsis thaliana), activator-inhibitor reaction-diffusion modeling has been proposed as a framework within which to interpret the known genetics [15, 26, 27]. The motivation was the publication by Gierer and Meinhardt [9] of their activatorinhibitor mechanism for biological pattern formation, since this mechanism directly yields a structure in which to interpret the formation of patterns, such as the positioning of trichomes, in terms of cellular and molecular processes. Their emphasis was on explaining how an activator-inhibitor mechanism could be the essential control of biological development and the associated pattern formation. Meinhardt subsequently explained how this concept could be applied to the modeling of specific situations [21] including pattern formation on shells [20] and in plants [19]. The application of the Gierer and Meinhardt activator-inhibitor interpretation to the positioning of trichomes from a genetic perspective followed $[13,15,26,27]$ once the developing molecular biology technology allowed for the easier identification of the genes controlling the differences between the wild type and mutants.

Motivation for the formulation of activator-inhibitor mechanisms has been the seminal publication of Turing [32] of a mathematical theory of chemical morphogenesis. Turing's conceptualization was profound because of its essential simplicity. His ansatz was that if, in the absence of diffusion, the reaction dynamics was such that its solution tended to a linearly stable uniform steady equilibrium, then, under appropriate conditions, the full reaction-diffusion system would generate spatially inhomogeneous patterns if the diffusion was destabilizing. The importance of Turing's 1952 paper, published the year before Watson and Crick's double helix paper [33], is that conditions were derived for which such destabilization would occur. The fact that such a process could generate patterns with a wide range of complexity which agreed with observed patterns in the physical and biological sciences became the stimulus for the subsequent explosion in reaction-diffusion research from both a practical [22] and theoretical perspective [12].

Remark. With respect to the above comments, it is appropriate to mention that reaction-diffusion partial differential equations first arose in the study of biological invasion and the nature of the dynamics being modeled there is quite different from that resulting from Turing's chemical morphogenesis theory. Biological invasion has an interesting history starting with the paper by Fisher in 1937 [8] and subsequently followed with different research contributions by Kolmogorov, Weinberger and others $[1,16,17,29,31,34]$.

Comment. The extent to which order and localization is an essential feature of the patterns that can be generated by reaction-diffusion systems has been examined from a number of independent perspectives. Lacalli [14] compared the patterns of cell wall growth in unicellular algae with a two morphogen reaction-diffusion model (Tyson's 
Brusselator) and concluded that the simulation of the growth could be viewed as a reaction-diffusion process. Holloway and Harrison [11] used the Clark and Evans $R$ parameter and the radial distribution function $g(t)$ to examine and characterize localization and order in reaction-diffusion patterns. Their discussion included a comparison of reaction-diffusion models with the inhibiting field concept of Wigglesworth [35]. Important as such publications are, they do not however examine mathematically the essential framework within which reaction-diffusion systems generate patterns and the limitations biologically that are thereby imposed.

As explained in Murray [22, Chapter 14], for a general two component system, which includes the Gierer and Meinhardt [9] activator-inhibitory mechanism as a special case, the essence of the associated mathematics involves the following steps:

(i) The Two-Component Reaction-Diffusion System.

On some two dimensional region $V$, the general non-dimensional form of a twocomponent reaction-diffusion system is given by

$$
\begin{array}{lll}
u_{t}=\gamma f(u, v)+\nabla^{2} u, & u=u(x, y, t), & u_{t}=d u / d t, \\
v_{t}=\gamma g(u, v)+d \nabla^{2} v, & v=v(x, y, t), & v_{t}=d v / d t,
\end{array}
$$

with zero flow conditions on the boundary $\partial V$ and given initial conditions on $V$

$$
(\mathbf{n} . \nabla)\left(\begin{array}{l}
u \\
v
\end{array}\right)=0, \quad u(x, y, 0), \quad v(x, y, 0) \text { given, }
$$

where $d$ denotes the ratio of the diffusion coefficients and $\gamma$ can be given various interpretations including being the ratio of the relative strengths of the reaction and the diffusion.

(ii) The Reaction Terms.

Depending on the application, the non-dimensional reaction terms $f(u, v)$ and $g(u, v)$ will take different forms. In terms of studied simple two-component systems, the applications include Schnakenberg's [28] two-species, chemical plausible, tri-molecular reaction, Thomas' real empirical substrate-inhibition system (Murray [22, Chapter 5]) and the activator-inhibitor mechanism of Gierer and Meinhardt [9]. That for Gierer and Meinhardt takes the form

$$
f(u, v)=a-b u+\frac{u^{2}}{v}, \quad g(u, v)=u^{2}-v, \quad a, b \text { constants. }
$$

Its special dynamics is the result of the interplay between the linear and quadratic terms driven by the autocatalytic term $u^{2} / v$. The other mentioned applications have a generically similar structure and thereby can be given an activator-inhibitor interpretation in terms of an interplay between linear and quadratic terms driven by an activator. 


\section{(iii) Linear Stability of Steady State.}

The pure reaction kinetics takes the following autonomous form (because no explicit spatial variation is involved in the definition of the reaction terms)

$$
u_{t}=\gamma f(u, v), \quad v_{t}=\gamma g(u, v) .
$$

Linearization of this equation about the steady state solution $\left(u_{0}, v_{0}\right)$ (i.e., the solution of the steady state equations $f(u, v)=0$ and $g(u, v)=0$ ) yields

$$
\mathbf{w}_{t}=\gamma A \mathbf{w}, \quad A=\left(\begin{array}{ll}
f_{u} & f_{v} \\
g_{u} & g_{v}
\end{array}\right)_{\left(u_{0}, v_{0}\right)}, \quad A \sim \text { stability matrix. }
$$

A standard analysis (cf. Murray [22], Section 14.3), which seeks the conditions for which

$$
\mathbf{w} \sim \exp (\lambda t)
$$

yields the following conditions on $\lambda$ which guarantee linear stability (i.e., $\operatorname{Re} \lambda$ $<0$ )

$$
\operatorname{trace}(A)=f_{u}+g_{v}<0, \quad \operatorname{determinant}(A)=f_{u} g_{v}-g_{u} f_{v}>0 .
$$

\section{(iv) Destabilizing Diffusion.}

On returning to the full reaction-diffusion system of equations (9.1), it is now necessary to perform a linear stability analysis of these equations about the steady state solution of this system. The details, not unexpectedly, are involved. However, in essence, it is now not only necessary to satisfy the condition (9.4) but also the conditions that arise in the stability analysis of the full system. Within the resulting set of constraints, the possibility occurs that instability can occur for just one of the wave numbers $k$ that defines the full solution of the linearized reaction-diffusion system. This leads naturally to the conclusion that a discrete pattern forms with the discreteness controlled by the relevant value of $k$.

For the reaction-diffusion modeling of pattern formation in plants, the connection to the associated biology and genetics is orchestrated via the assumption that the molecular dynamics controlling the pattern formation is an activator-inhibitor mechanism. This then imposes the constraint that the known genes involved must be interpreted from this perspective. In this mechanism, the activator tries to find a point in the pattern domain where it can locally dominate the inhibitor. At such a point, the pattern feature (e.g., trichome) will arise. The local nature of the dominance of the activator does not allow more than one activator to be present near the pattern feature activation point, and this in turn limits clumping effects.

Though such modeling is able to reproduce some of the data for trichome positioning, it has a number of drawbacks:

(i) The pattern arises as an instability in the reaction-diffusion process which is difficult to explain biologically. 
(ii) The control of its parameterization to produce mutants is problematic and it is hard to interpret them biologically.

(iii) It is challenged to reproduce the clumping mutant.

(iv) The reaction-diffusion equations model an outward moving wave which is not consistent with the assumption that the fate of the leaf is decided at the meristem.

An alternative modeling concept, which captures cell communication and allows clumping effects, is proposed in the next section.

\subsubsection{Hexagonal Recursion}

The following discussion of hexagonal recursion and its application to modeling the positioning of trichomes on the leaves of plants is based on the earlier deliberations of [7].

The basic assumption on which that modeling was based is that the fate of the cells on the leaf of a plant is determined as it grows out of the meristem as illustrated in Figure 9.1 (a). The structure of the epidermal surface of a leaf is assumed to take the form of a hexagonal array. This is in keeping with the results in Table 1 of [15], where the average number of sides of the cells is approximately six. In general, plant cells are not hexagonal, however, the cells do tend to have a structure that is topologically similar to a hexagonal array. For example, a running rectangular brick array is topologically equivalent to a hexagonal array.

It is assumed that the concentration of the signal controlling whether a cell becomes a trichome accumulates according to the additive rule of Figure 9.1 (b). In the sequel, this formula will be referred to as the "hexagonal recursion". The numerical values generated by this recursion will be referred to as "hexagonal concentration values" (HCV).

For this model, a number of important biological constraints are automatically taken into account:

(i) The model is cellular, and not some macroscopic model that has smoothed out the cellular details.

(ii) The fate of a cell is determined by its neighbors [24].

(iii) As implied in various papers by Wolpert such as [36], any cellular model must respect the known positional information behavior in the biological development of that part of an organism that is being modeled.

(iv) Because of the directional nature of the hexagonal recursion, in defining how the concentration of the key signal accumulates, the model automatically involves a polar transport mechanism.

(v) Trichomes do not form in boundary cells of an Arabidopsis leaf [26]. 


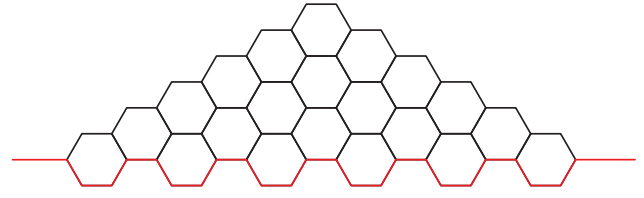

(a)

Meristem

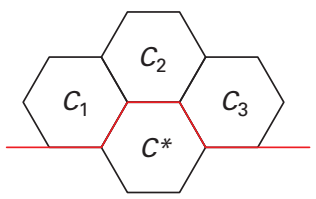

(b) $\quad C^{*}=w_{l} C_{1}+w_{c} C_{2}+w_{r} C_{3}$

Figure 9.1. (a) A hexagonal cell approximation of the epidermal cells on the upper side of a leaf. The top hexagonal cell corresponds to the tip of the leaf. The red line represents the meristem out of which the leaf is growing. Further details can be found in [25]. (b) The localized additive relationship that models how the concentration of the signal in the cells above the meristem determine its concentration in the cells forming at the meristem.

In Section 9.1.3.1, the hexagonal recursion is defined. It must be robust in that small changes in the value of the defining parameters give only small changes in the patterns generated. This does not rule out the fact that large changes in the parameter will give large changes in the patterns. In Section 9.1.3.2, patterns are generated which are of wild type. In Section 9.1.3.3, by varying a particular parameter in the hexagonal recursion, an extensive range of synthetic mutants is generated.

\subsubsection{The Hexagonal Recursion Rule}

The hexagonal recursion rule for determining the $\mathrm{HCV}$ of a cell and the consequential positioning of trichomes is given by:

R1. Tiles located on the periphery of the leaf have the "boundary" $\mathrm{HCV} P_{0}$.

R2. The leaf starts growing from the three tip hexagons in the manner indicated in Figure 9.1 (b). The HCV $C^{*}$ of the cell forming at the meristem is determined by

$$
C^{*}=w_{l} C_{1}+w_{c} C_{2}+w_{r} C_{3}, \quad w_{l} \geq 0, w_{c} \geq 0, w_{r} \geq 0,
$$

where $w_{l}+w_{c}+w_{r} \geq 1$, which guarantees that, until reset, the HCV increases in the cell(s) forming at the meristem.

R3. This process continues progressively for each triple of hexagonal cells above the meristem as the leaf step-by-step grows out of the meristem.

R4. With respect to a specified threshold $T_{1} \geq P_{0}$, if a cell, forming at the meristem as indicated in Figure 9.1 (b), has $\mathrm{HCV} C^{*} \geq T_{1}$, then the fate of that cell is set to be a trichome.

R5. With respect to a specified threshold $T_{2} \geq T_{1}$, if, in a triple of hexagonal cells above the one that they are involved in forming at the meristem, at least one of them has HCV greater than or equal to $T_{2}$, then the $\mathrm{HCV} C^{*}$ is reset to be $P_{0}$. 
Two trivial asymptotic situations can arise from this hexagonal recursion. When the first threshold value $T_{1}=P_{0}$, the boundary value, and the second threshold value $T_{2} \rightarrow \infty$, the ON threshold is always met and the OFF threshold is never met, and so trichomes are produced in every cell, including the boundary. The other trivial case arises when the threshold value $T_{1}$ is so high that it is never met and so no trichomes are ever activated.

\subsubsection{Wild Type Patterns}

The recursion rule is used with the OFF threshold value $T_{2}$ equal to the $\mathrm{ON}$ threshold value $T_{1}$. This means that as soon as a cell reaches the threshold value to produce a trichome, any adjacent cells below have their value reset to the initial value $P_{0}$, and the possibility of a trichome is "switched off".

In Figures 9.2 and 9.3, the HCVs have been determined according to the hexagonal recursion. The colour of the cell represents the corresponding HCV with white corre-

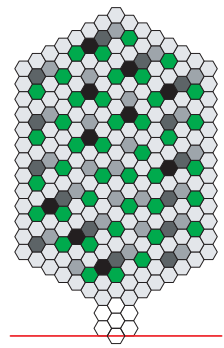

(a) $\quad T_{1}=20$

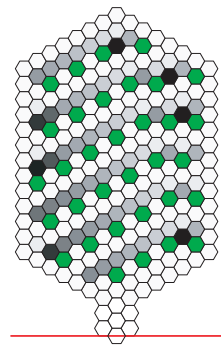

(b) $T_{1}=100$

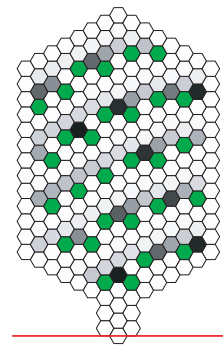

(c) $T_{1}=500$

Figure 9.2. Final distributions generated by the hexagonal recursion with $T_{1}=T_{2}$ and (a) $T_{1}=20$, (b) $T_{1}=100$, (c) $T_{1}=500$, with weights $w_{l}=4, w_{c}=2, w_{r}=1$ and boundary value $P_{0}=1$. (Note that the shading changes as the value of $T_{1}$ changes.)

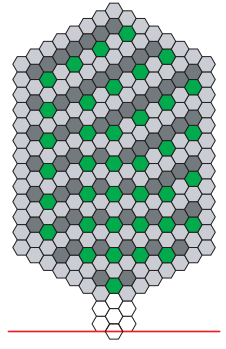

(a) $\quad T_{1}=10$

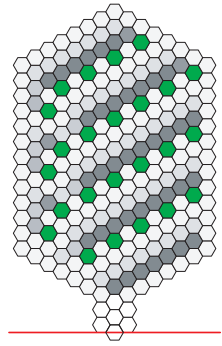

(b) $\quad T_{1}=50$

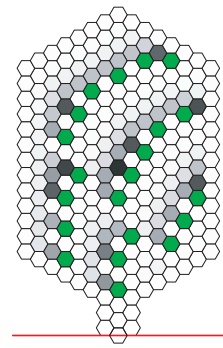

(c) $T_{1}=500$

Figure 9.3. Final distributions generated by the hexagonal recursion with $T_{1}=T_{2}$ and (a) $T_{1}=10$, (b) $T_{1}=50$, (c) $T_{1}=500$, with weights $w_{l}=4, w_{c}=1, w_{r}=0$ and boundary value $P_{0}=1$. 
sponding to zero and black to the maximal value one less than the threshold value $T_{1}$ (i.e. $\left.T_{1}-1\right)$. The cells which become trichomes where the $\mathrm{HCV}$ matches or exceeds the threshold value $T_{1}$ are marked green.

It can be shown that if symmetric weights (i.e., $w_{l}=w_{r}$ ) are chosen, symmetric patterns will be produced. These types of patterns are not presented and all cases illustrated have non-symmetric weights $w_{l} \neq w_{r}$. Even so, artifactual, symmetric-like patterns (e.g., Figure 9.3 (a)) can form. It is also possible to generate diagonal patterns (e.g., Figure 9.2 (b) and $9.3(\mathrm{~b})$ ).

In Figure 9.2, the value of the switching thresholds $T_{1}\left(=T_{2}\right)$ are increased with all other parameter values fixed. This leads to a delay in the appearance of cells producing trichomes. Increasing the value of $T_{1}$ (and $T_{2}$ ) will lead to the first trichome production located further down the leaf. This pattern is also observed in Figure 9.3. From Figures 9.2 and 9.3, it can also been seen that as the threshold value $T_{1}\left(=T_{2}\right)$ increases, the number of cells producing trichomes decreases and the pattern becomes more sparse. Further increases in $T_{1}$ will result in less trichome production, with the limiting case of no trichome production occurring as $T_{1} \rightarrow \infty$.

\subsubsection{Mutant Patterns}

As is clear from the discussion in Section 9.1.3.2 there is no possibility of generating clumps of trichomes when $T_{2}=T_{1}$, since as soon as a cell reaches the threshold value to produce a trichome, any adjacent cells below have their value reset to the initial value $P_{0}$, and the possibility of a trichome is "switched off". Allowing the HCV $C^{*}$ in a cell to have neighboring cells that have produced a trichome but have not reached or exceeded the second, higher threshold will allow neighboring cells to become trichomes. This establishes that two distinct thresholds are required to produce clumping and, consequently, parameter choices with $T_{2}>T_{1}$ are required to generate mutants.

Figures 9.4 and 9.5 both have $w_{l} \neq w_{r}$ and the resulting patterns are generally not symmetric. It is obvious that clumping of trichomes is possible in the hexagonal re-

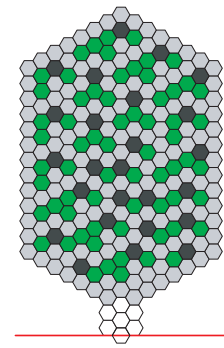

(a) $T_{2}=20$

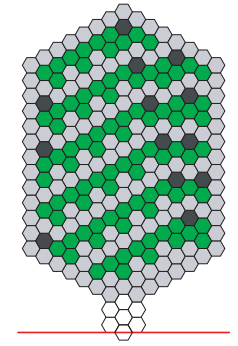

(b) $T_{2}=100$

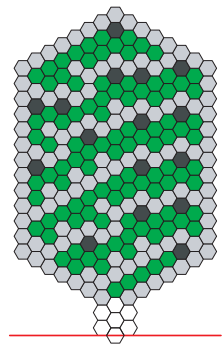

(c) $T_{2}=300$

Figure 9.4. Final distributions generated by the hexagonal recursion with $T_{1}=10$ and (a) $T_{2}=20$, (b) $T_{2}=100$, (c) $T_{2}=300$, with weights $w_{l}=4, w_{c}=2, w_{r}=1$ and boundary value $P_{0}=1$. 


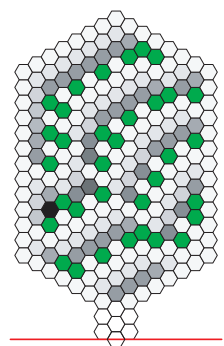

(a) $T_{2}=100$

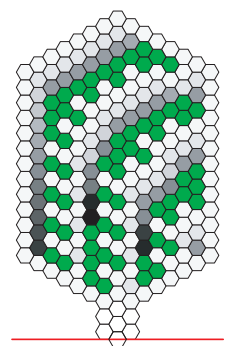

(b) $T_{2}=400$

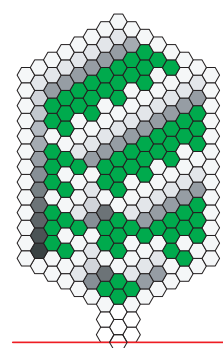

(c) $T_{2}=800$

Figure 9.5. Final distributions generated by the hexagonal recursion with $T_{1}=60$ and (a) $T_{2}=100$, (b) $T_{2}=400$, (c) $T_{2}=800$, with weights $w_{l}=4, w_{c}=1, w_{r}=0$ and boundary value $P_{0}=1$.

cursion model when $T_{2}>T_{1}$. Holding the threshold value $T_{1}$ fixed and increasing the value of $T_{2}$ leads to a thickening of the groups of cells producing trichomes. Increasing the threshold value $T_{1}$ will result in the initial production of trichomes occurring further down the leaf. Setting the right-hand weight $w_{r}=0$ leads to a trichome pattern which is pushed toward the right-hand side of the leaf.

\subsubsection{Conclusions}

As conjectured in Pereverzyev and Anderssen [25], having a notional model of the type formulated above, yields a new framework for performing the biocombinatorial sorting of the known genes to be involved into biomechanistic categories. The traditional approach, as exemplified in Digiuni et al. [6], is basically biological as the modus operandi is driven by comparative genetics based on differences in phenotype between mutants and wild type. Appealing to a complex mathematics differential equation model is of little assistance unless that model has or can be related explicitly to the genetic/biological processes occurring. In addition, the formulation of a differential equation model that simulates the observed biological dynamics, though interesting and informative, is not a proof of or framework for the analysis of the biomechanistic dynamics occurring.

An alternative strategy, which is being proposed here, is the formulation of simple models, defined in terms of variables which can be given specific biological meaning (e.g., the concentrations of key hormones), which yield a mechanistic framework within which the comparative genetics is performed. As already mentioned, the earlier pea leaf modeling of Young [38] represents an excellent model system of the process being proposed. A notional protocol for doing this in the context of the positioning of trichomes on the leaves of plants can be found in Section 5 of Pereverzyev and Anderssen [25]. 
Acknowledgments. The authors are grateful to the referees whose comments have assisted in the improvement of this chapter. Publications by the authors related to earlier versions of this research have been acknowledged in the text. This chapter represents an extension of and further development of their ideas in their MODSIM 2011 Conference paper entitled "Modelling pattern formation in plants".

\section{Bibliography}

[1] D. G. Aronson, H.F. Weinberger, Multidimensional non-linear diffusion arising in population-genetics, Advances in Math. 30 (1978), 33-76.

[2] E. Coen, The Art of Genes, Oxford University Press, Oxford, 1999.

[3] E. Coen, A. G. Rolland-Lagan, M. Matthews, J. A. Bangham, P. Prusinkiewicz, The genetics of geometry, PNAS, 101 (2004), 4728-4735.

[4] M.-L. Cui, L. Copsey, A. A. Green, J. A. Bangham, E. Coen, Quantitative control of organ shape by combinatorial gene activity, PLOS Bio. 8 (2010), e1000538.

[5] F. R. de Hoog, Why are simple models often appropriate in industrial mathematics? in: 18th World IMACS/MODSIM Congress, Cairns, July 13-17, Proceedings (2009), 23-36.

[6] S. Digiuni, S. Schellmann, F. Geier, B. Greese, M. Pesch, K. Wester, B. Dartan, V. Mach, B. P. Srinivas, J. Timmer, C. Fleck, M. Hulskamp, A competitive complex formation mechanism underlies trichome patterning on Arabidopsis leaves, Mol. Systems Bio. 4, Article Number 217 (2008).

[7] M. P. Edwards, S. Pereverzyev Jr., R. S. Anderssen, Modelling pattern formation in plants, in: MODSIM 2011 Congress, Perth, December 12-16, Proceedings (2011), 378384.

[8] R. A. Fisher, The wave of advance of advantageous genes, Annals Eugenics 7 (1937), 355-369.

[9] A. Gierer, H. Meinhardt, Theory of biological pattern formation, Kybernetik 12 (1972), 30-39.

[10] A. A. Green, R. Kennaway, A. I. Hanna, J. A. Bangham, E. Coen, Genetic control of organ shape and tissue polarity, PLOS Biology 8, Article Number el000537 (2010).

[11] D. M. Holloway, L. G. Harrison, Order and localization in reaction-diffusion pattern, Physica A - Stat. Mech. and Appl. 222 (1995), 210-233.

[12] R. B. Hoyle, Pattern Formation: An Introduction to Methods, Cambridge University Press, Cambridge, 2006.

[13] M. Hulskamp, Plant trichomes: A model for cell differentiation, Nature Rev. Molecular Cell Biol. 5 (2004), 471-480.

[14] T. C. Lacalli, Dissipative structures and morphogenetic pattern in unicellular algae, Phil. Trans. Roy. Soc. London - B. Bio. Sciences 294 (1981), 547-588.

[15] J. C. Larkin, N. Young, M. Prigge, M. D. Marks, The control of trichome spacing and number in Arabidopsis, Development 122 (1996), 997-1005. 
[16] B. T. Li, M. A. Lewis, H. F. Weinberger, Existence of traveling waves for integral recursions with nonmonotone growth functions, J. Math. Biology 58 (2009), 323-338.

[17] B. T. Li, H. F. Weinberger, M. A. Lewis, Spreading speeds as slowest wave speeds for cooperative systems, Math. Biosci. 196 (2005), 82-98.

[18] A. Lindenmayer, Developmental algorithms for multicellular organisms-Survey of L-systems, J. Theor. Biology 54 (1975), 3-22.

[19] H. Meinhardt, Models of pattern formation and their application to plant development, in: P. W. Barlow and D. J. Carr (eds.), Positional Control in Plant Development Chapter 1, Cambridge University Press, Cambridge, (1984), 1-32.

[20] H. Meinhardt, M. Klingler, A model for pattern-formation on the shells of mollusks, $J$. Theor. Bio. 126 (1987), 63-89.

[21] H. Meinhardt, Models of biological pattern formation: From elementary steps to the organization of embryonic axes, in: S. Schnell, P. K. Maini, S. A. Newman, T. J. Newman, (eds.), Multiscale Modelling of Developmental Systems, Current Topics in Developmental Biology 81, pp. 1-63, 2008, 9th Biocomplexity Workshop, Bloomington, IN, MAY, 2006.

[22] J. D. Murray, Mathematical Biology, Springer, Berlin, 1989.

[23] C. M. O'Keefe, S. Pereverzyev Jr., R. S. Anderssen, The algebra of hexagonal numbers, The Mathematical Scientist 36 (2011), 1-9.

[24] R. I. Pennell, Q. C. B. Cronk, S. Forsberg, C. Stohr, L. Snogerup, P. Kjellbom, P. F. McCrae, Cell-Ccntex signalling, Phil. Trans Roy. Soc. London, B-Biological Sci. 350 (1995), 87-93.

[25] S. Pereverzyev Jr., R. S. Anderssen, Recursive algebraic modelling of gene signalling, communication and switching, RICAM Report 24 (2008), 17.

[26] S. Schellmann, A. Schnittger, V. Kirik, T. Wada, K. Okada, A. Beermann, J. Thumfahrt, G. Jurgens, M. Hulskamp, TRIPTYCHON and CAPRICE mediate lateral inhibition during trichome and root hair patterning in Arabidopsis, EMBO J. 21 (2002), 5036-5046.

[27] B. Scheres, Plant patterning: TRY to inhibit your neighbors, Current Bio. 12 (2002), R804-R806.

[28] J. Schnakenberg, Simple chemical-reaction systems with limit-cycle behavior, $J$. Theor. Biol. 81 (1979), 389-400.

[29] N. Shigesada, K. Kawasaki, Biological Invasion: Theory and Practice, Oxford University Press, Oxford, 1997.

[30] D. Taimina, Crocheting Adventures with Hyperbolic Planes, A. K. Peters Ltd., Wellesley, MA, 2009.

[31] H. R. Thieme, Density-dependent regulation of spatially distributed populations and their asymptotic speed of spread, J. Math. Biology 8 (1979), 173-187.

[32] A. M. Turing, The chemical basis of morphogenesis, Phil. Trans. R. Soc. London Ser. B-Biol. Sci. 237 (1952), 37-72. 
[33] J. D. Watson, F. H. C. Crick, Molecular structure of nucleic acids - A structure for deoxyribose nucleic acid, NATURE 171 (1953), 737-738.

[34] H. F. Weinberger, Long-time behavior of a class of biological models, SIAM J. Math. Anal. 13 (1982), 353-396.

[35] V. B. Wigglesworth, Local and general factors in the development of "pattern" in Rhodnius prolixus (hemiptera), J. Exp. Bio. 17 (1940), 180-200.

[36] L. Wolpert, Positional information and spatial pattern of cell differentiation, J. Theoretical Biol., 25 (1969), 1-47.

[37] L. Wolpert, The Development of Pattern and Form in Animals, Carolina Biology Readers, No. 51, Carolina Biological Supplies, Burlington, NC, (1977), 1-16.

[38] J. P. W. Young, Pea leaf morphogenesis - a simple-model, Annals Botany 52 (1983), 311-316.

\section{Author Information}

Robert S. Anderssen, CSIRO Mathematics, Informatics and Statistics, Canberra, Australia

E-mail: Bob.Anderssen@csiro.au

Maureen P. Edwards, School of Mathematics and Applied Statistics, University of Wollongong, Wollongong, Australia

E-mail: maureen@uow.edu . au

Sergiy Pereverzyev Jr., Industrial Mathematics Institute, Johannes Kepler University Linz, Linz, Austria

E-mail: pereverzyev@indmath .uni-linz.ac.at 